\title{
LA EDUCACIÓN SUPERIOR EN AMÉRICA LATINA: ¿PROBLEMAS INSOLUBLES O RECETAS INADECUADAS?
}

\author{
Adriana Chiroleu*
}

Recebido: 02 fev. 2011

Aprovado: 18 maio 2011

* Doctora en Ciencias Sociales por FLACSO y la Universidad de Brasilia, Profesora Titular de la Facultad de Ciencia Política y Relaciones Internacionales de la Universidad Nacional de Rosario e Investigadora Independiente del CONICET / Argentina. E-mail: achiroleu@arnet.com.ar

Resumen: Este texto presenta algunas tendencias comunes en el ámbito regional y varios de los principales nudos problemáticos que obstaculizan un desarrollo armónico de su educación superior. Consideramos al respecto, que la continuidad de las políticas públicas pergeñadas en los 90 , ha bloqueado la posibilidad de resolver problemas medulares. Focalizaremos especialmente en tres procesos: la expansión de la cobertura sin reducción de las desigualdades sociales ni inclusión plena en la educación superior, el desarrollo de procesos de evaluación institucional sin mejoramiento efectivo de la calidad de los aprendizajes y el énfasis otorgado a la formación de profesionales que subalterniza el compromiso y la responsabilidad social de los universitarios.

Palabras clave: Políticas de educación superior . América Latina. Evaluación. Inclusión.

\author{
HIGHER EDUCATION IN LATIN AMERICA: \\ UNSOLVABLE PROBLEMS OR MISLEADING RECIPES?
}

\begin{abstract}
This paper points out some common tendencies of the Latin America region and several troublesome aspects that hinder a harmonic development of its Higher Education. We consider that the sustainability of the public policies sketched out in the ' $90 \mathrm{~s}$, has blocked the possibility of solving major problems. We will pay extra attention to the three following processes: the expansion of the outreach without the reduction of either social inequalities or a fully inclusion in Higher Education; the development of institutional evaluation processes without an improvement on the quality of the learning processes; and last but not least, the emphasis given to professional trainings instead of the commitment and the social responsibility of the university actors.
\end{abstract}

Key words: Higher education policies. Latin America. Evaluation. Inclusion.

La primera década del siglo XXI ha sido testigo de una importante renovación política en América Latina, en la que varios países asisten a nuevas experiencias de centroizquierda que - denominadas genéricamente "progresistas"- se presentan como alternativa a las gestiones neoliberales de los años 90. El énfasis que éstas pusieron en la reestructuración del estado y la plena vigencia de las leyes de mercado generó un crecimiento de la subutilización 
de la fuerza de trabajo y profundizó la pobreza a partir del desentendimiento del estado de sus responsabilidades en ámbitos tan sensibles como la salud, la educación y la seguridad social.

Estas reformas alcanzaron también a la educación superior resultando por entonces profundamente transformada a través del establecimiento de cuerpos intermedios de control y coordinación y programas especiales, que mudaron en poco tiempo su fisonomía.

En lo que va del siglo XXI, y en el contexto de gobiernos de signo diverso y de un reconocimiento de los claroscuros de los 90, los altos precios internacionales de los productos de exportación generaron mejores condiciones económicas y sociales iniciándose una etapa caracterizada por un crecimiento del PBI, y un mejor comportamiento de las principales variables sociales: reducción del desempleo, disminución de la pobreza e indigencia, aumento de los salarios reales, etc. aunque la desigualdad y la fragmentación social siguen constituyendo una marca persistente en la región. En este nuevo clima, era esperable que la renovación introducida en diversos ámbitos de las políticas públicas alcanzara también a la educación superior.

Aún reconociendo las dificultades que entraña la pretensión de abrir espacios de reflexión en torno a América latina, esta presentación procurará reseñar algunas tendencias comunes en el ámbito regional y varios de los principales nudos problemáticos que obstaculizan un desarrollo armónico de su educación superior. En este sentido, consideramos que la continuidad de las políticas públicas pergeñadas en los 90 , que en algunos países ya conforman políticas de estado, ha bloqueado la posibilidad de resolver / contener problemas medulares.

En este contexto general, se presentan, aunque con diverso énfasis, en casi todos los países procesos de doble cara que dan cuenta de transformaciones inacabadas o inconclusas que tienden a potenciar las "marcas de nacimiento" de la educación superior de América Latina. Los mismos, invocando como meta la modernización de los sistemas, no hacen más que acentuar los rasgos más regresivos de los mismos. En este trabajo focalizaremos especialmente en tres procesos que sin ser los únicos, constituyen sin embargo tres problemáticas de vasto alcance: la expansión de la cobertura sin reducción de las desigualdades sociales ni inclusión plena en la educación superior, el desarrollo de procesos de evaluación institucional sin mejoramiento de la calidad de los aprendizajes y el fuerte énfasis otorgado a la formación de profesionales por sobre el compromiso y la responsabilidad social universitaria. 


\section{LAS SINGULARIDADES DE LAS POLIITICAS DE EDUCACIÓN SUPERIOR ${ }^{1}$}

Una revisión de las características de la política de educación superior en el conjunto de las políticas públicas de los 90, da cuenta que en el auge de la estrategia neoliberal, éste sector estuvo sometido a políticas que en lugar de reducir, aumentaron el control del estado con el fin de ejercer una regulación más firme sobre las instituciones y el sistema de educación superior. Entre los académicos no existe sin embargo, acuerdo sobre la forma de rotular este proceso que la mayoría designa sin matices como neoliberal, otros califican como "neo-intervencionismo" (ACOSTA SILVA, 2002), mientras otros ponen el énfasis en el carácter neoconservador de la intervención en el ámbito de la educación superior y aún, en la carga modernizadora de las nuevas políticas. Lo cierto es que, si la tónica de la época fue reducir / achicar el estado, en la educación superior, el signo fue inverso, y el estado fortaleció su presencia regulatoria a través de la creación de diversas instituciones y mecanismos.

¿Cómo entender esta paradoja?, ¿cómo se explica el signo diverso de la política en este sector? Podría argumentarse que no se trata de una paradoja pues el estado se constituyó por esos años en garante de la aplicación de los postulados de los organismos multilaterales de crédito, y procuró por esta vía reducir los aportes que el erario público efectuaba al sector. Esta situación, puede hipotetizarse, se relaciona con los efectos de los procesos de globalización e internacionalización de la educación superior y el lugar que ocupan en el mismo los países periféricos. Esto supone reconocer, más allá de la retórica, un lugar subalterno para la educación superior nacional en vista de los requerimientos planteados en el orden internacional. ${ }^{2}$

En último término, se trataría de un reconocimiento de la singularidad del campo de la educación superior, un ámbito tradicionalmente protegido por los estados, que requiere la generación de condiciones especiales que conduzcan a un ingreso pleno del mercado.

En el contexto de la primera década del siglo XXI, y a pesar de la renovación política experimentada en buena parte de los países y del reconocimiento del fracaso de las políticas encaradas por el neoliberalismo, una vez más, la política aplicada a la educación superior no sigue los mismos carriles que la

1 Este trabajo se encuadra en un proyecto de investigación más amplio sobre Políticas de Educación Superior en América Latina en el siglo XXI, financiado por el CONICET, y que aborda específicamente los casos de Argentina, Brasil, México y Venezuela. Por ese motivo, se ejemplificará especialmente a través de información proveniente de estos países, lo cual no implica desconocer que en algunas temáticas, otros casos nacionales resultan más representativos.

2 Véase al respecto entre otros trabajos, Ordorika (2006) y Santos (2007). 
que se desarrolla en otros sectores. Al punto que se plantean fuertes líneas de continuidad con las políticas de los 90 , o en todo caso, se trata de un espacio en el cual la voluntad de renovación, al menos en varios de los países, resulta limitada. En este sentido, la evaluación continúa siendo un eje articulador de la política sectorial, y el desarrollo de políticas inclusivas de diversa índole según las características de los casos nacionales es abordada como mecanismo para mitigar (y no para atender proactivamente) las bajas tasas de cobertura que existen en numerosos países y las desiguales oportunidades de acceso -y sobre todo de graduación- que los diversos grupos sociales tienen en todos.

La ampliación del acceso a la educación superior es reconocida como una vía privilegiada para mejorar los ingresos en una sociedad, y operar sobre las desigualdades sociales, factores potenciales de ampliación de la democratización social y expansión de la ciudadanía. Se trataría en este caso de reconocer que más allá de las funciones clásicas de la universidad en términos de producción y reproducción de la cultura, existen otros aportes ligados a la generación de movilidad social ascendente, especialmente importantes en sociedades profundamente desiguales como las latinoamericanas, en las que las posiciones sociales se encuentran, en gran medida, cristalizadas ${ }^{3}$.

Convendría sin embargo, más allá de las generalidades, singularizar la situación de los países de la región. Si tomamos por ejemplo, los casos de Argentina, Brasil, México y Venezuela, en el transcurso de las gestiones de gobierno del siglo XXI, podríamos ubicarlos en un continuo que, extendido entre los polos renovación-continuismo, da cuenta de Venezuela y México, ocupando respectivamente los extremos y de Brasil y Argentina, en las posiciones intermedias.

El caso de Venezuela presenta aristas especiales en la medida en que, se da un cambio de paradigma en el desarrollo universitario y las políticas se articulan a partir de un nuevo eje, el de la inclusión ${ }^{4}$. En este caso, sí puede plantearse una renovación a partir de un interés expreso del gobierno de Chávez de incidir en el sector y ampliar la cobertura incorporando a segmentos de la población tradicionalmente alejados de este nivel educativo. Este proceso se da sin embargo, en instituciones nuevas, creadas ad hoc y de manera vertiginosa, que generan una fuerte expansión de la escolarización superior. ${ }^{5}$ Se ha conformado

3 Sobre esta temática puede consultarse Acosta Silva (2002).

4 Escogemos el caso venezolano por constituir en el contexto regional del siglo XXI una situación especial de transformación de las políticas y los paradigmas universitarios. Es necesario reconocer sin embargo, que especialmente en las políticas de expansión de la cobertura e inclusión, el mismo está estrechamente vinculado a políticas y prácticas desarrolladas en Cuba en las últimas décadas que han redundado en que este país tenga -según datos UNESCO correspondiente al año 2010- una TBES del orden del $88 \%$ de los jóvenes entre 18 y 24 años.

5 Según datos provistos por el Ministerio del Poder Popular para la Educación Superior, en el año 2000, 
sin embargo, un circuito institucional paralelo al tradicional y se presentan dudas razonables sobre la calidad de los aprendizajes, lo que puede redundar en una reducción de los efectos democratizadores de las políticas inclusivas ensayadas.

En el polo opuesto, la evaluación ha pasado en México -según lo expresan los propios documentos oficiales- a conformar una política de estado, sin que en este proceso mediara una revisión crítica de sus luces y sombras. Paralelamente se han incrementado en cantidad y se han ampliado en abarcatividad las políticas de inclusión en procura de mejorar las Tasas Brutas de Escolarización Superior (TBES), cuestión ésta que comienza a ser reconocida como un área problemática.

En posiciones intermedias dentro del continuo pueden ubicarse los casos de Brasil y Argentina. En el primer caso, se introducen en la educación superior políticas inclusivas de vasto alcance, pero tanto éstas, como las de evaluación parecen quedar sometidas a la capacidad de lobby de ciertos grupos con alto poder de incidencia.

En Argentina, por su parte, la educación superior parece subordinada a demandas más urgentes originadas en otros sectores de la vida política y social. Si bien la retórica oficial da cuenta de la sustitución de la concepción de calidad propia de los 90, ligada a la búsqueda de eficiencia, para asociarla más con la idea de mejoramiento, el encuadre legal, los documentos y las pautas técnicas que permiten su aplicación siguen siendo las mismas que se gestaron promediando aquella década.

Dentro de la continuidad de estas políticas se inscribe el fracaso de la universidad para resolver algunos de sus problemas medulares: la ampliación de la cobertura sin generación de un proceso inclusivo ni reducción de las desigualdades sociales, el desarrollo ritual de procesos de evaluación institucional sin mejoramiento efectivo de la calidad y la opción por una formación profesionalista de los universitarios que los aleja de posturas más comprometidas con la realidad social.

\section{EXPANSIÓN DE LA COBERTURA SIN REDUCCIÓN DE LAS DESIGUALDADES SOCIALES NI INCLUSIÓN PLENA EN LA EDUCACIÓN SUPERIOR}

En las últimas décadas se ha dado en América latina una expansión constante de la cobertura en educación superior cuya matrícula supera en la actualidad 
los 16 millones de estudiantes, con una TBES próxima al 30\% de la población entre 20 y 24 años. Este dato, por sí mismo, no alcanza a dar cuenta de un proceso por demás de complejo y paradójico, en el que es necesario destacar por una parte, la importancia de la expansión la cual ha constituido además un rasgo constante e independiente de las crisis cíclicas que atraviesa la región. Sin embargo, la lectura del dato resulta menos optimista si se lo contrasta con el correspondiente a los países centrales, en los cuales dicha tasa es prácticamente el doble ${ }^{6}$ y si se reconoce además su carácter de promedio regional que esconde profundas diferencias entre los países: al respecto, Bolivia, Chile y Uruguay se ubican por encima de ese promedio y Argentina, Cuba y Venezuela, superan incluso el 50\% de cobertura; en el otro extremo algunos de los sistemas más grandes de la región se ubican por debajo del promedio. ${ }^{7}$

Este reducido nivel de cobertura en la región se presenta acompañado además por altas tasas de deserción y una acentuación de la segmentación de los sistemas, los cuales quedan conformados por circuitos institucionales de objetivos diversos, muchos de los cuales se orientan especialmente a atender intereses particularistas. En todos los casos se verifica además, una valoración utilitarista de los procesos educativos y de las titulaciones las cuales acentúan la búsqueda de éxito individual (LANDINELLI, 2008).

En este mismo sentido, el segmento privado de la educación superior se expande, alcanzando a cubrir en promedio alrededor del $45 \%$ de la matrícula total: los extremos son, países que superan el $70 \%$ como Brasil y Chile y otros que no alcanzan el $20 \%$, como Uruguay y Bolivia. Argentina ${ }^{8}$ y Venezuela están apenas por encima del $25 \%$, mientras que México se ubica en una situación intermedia y alcanza el $33 \%$.

Dentro del sector privado también prima la heterogeneidad y entre los establecimientos se destaca un segmento de excelencia orientado a captar la demanda de los hijos de las élites sociales y políticas, el cual coexiste con otro que procura atender una demanda excedente que, por diversos motivos, no se canaliza a través de las instituciones tradicionales. Entre ambos segmentos las diferencias en términos de calidad y de posibilidades de inserción en el mercado ocupacional, son abismales.

6 En este sentido, según datos de UNESCO, Estados Unidos y Europa occidental tienen en promedio una TBES del orden del $57 \%$ (DIDRIKSON, 2008).

7 México tiene una TBES que aún no alcanza el 27\% y Brasil se ubica en el orden del 18\%.

8 En Argentina, los últimos años han mostrado una fuerte reducción del ritmo de expansión de la matrícula total; si dentro de ésta se considera su componente público y privado, puede observarse que el índice de crecimiento de éste (29\%) está muy por encima del de aquel (1\%).

9 El segmento privado constituía sin embargo, el 23\% hacia mediados de los años 90, por lo que la expansión de los últimos quince años, ha sido sostenida. 
Desde la perspectiva social, existe una sobre-representación de los sectores sociales más acomodados y una reducida presencia de los grupos socioeconómicos menos favorecidos ${ }^{10}$; por otra parte, entre los que efectivamente ingresan, se dan disímiles posibilidades de concluir con éxito el tránsito por el tercer nivel y obtener así el grado académico respectivo. Esto es, en buena medida se sigue promoviendo el ingreso formal a las instituciones, sin generar condiciones para que los grupos más desfavorecidos, puedan no sólo atravesar la puerta de ingreso y estacionarse transitoriamente en las instituciones, sino, efectuar aprendizajes relevantes y obtener el diploma en las mismas condiciones que sus compañeros de sectores más favorecidos.

La noción de inclusión ${ }^{11}$ incorporada recientemente al léxico de la educación superior, parte del reconocimiento que la sociedad no es homogénea y la diversidad constituye un componente fundamental que merece ser revalorizado; al ser aplicada a este ámbito desde una perspectiva amplia, propone integrar los conceptos de equidad de acceso, participación, aprovechamiento, progreso y culminación de los estudios. Estos procesos deberían estar acompañados por la generación de condiciones de aprendizaje que tengan en cuenta las necesidades de los grupos tradicionalmente excluidos y sus niveles de rezago en capacidad cognitiva y conocimiento para operar efectivamente en la reducción de la desigualdad y el aumento de la cohesión social (APONTE HERNÁNDEZ, 2008, p. 20).

En los últimos años se han puesto en marcha políticas tendientes a contemplar esta problemática que procuran complementar el clásico apoyo económico a estudiantes carenciados; entre ellas las políticas de acción afirmativa (cuotas para minorías étnico-raciales, cursos especiales para esos grupos, etc.) y la creación de nuevas instituciones (especialmente las universidades indígenas/ interculturales) constituyen experiencias novedosas.

$\mathrm{Al}$ respecto, aunque constituyen un progreso significativo, las mismas aún focalizan mayoritariamente en la ampliación de las posibilidades de acceso a la educación superior, sin poner igual énfasis en la atención de las problemáticas ulteriores. Por otra parte, la segmentación que opera en los sistemas limita las posibilidades de reducir la incidencia de los factores socioeconómicos.

10 El promedio regional indica que el $50 \%$ de los hijos del quintil más alto de ingresos desarrolla estudios superiores, mientras sólo lo hace menos del $20 \%$ de los hijos del quintil más pobre (APONTE HERNÁNDEZ, 2008). En los casos de México y Brasil, la situación es más alarmante pues los dos quintiles inferiores prácticamente no consiguen ingresar, mientras el quintil más rico se aproxima a la universalización de la cobertura. La TBES del quintil más rico es mayor al 90\% en Brasil y se ubica en el orden del $75 \%$ en México, mientras los dos quintiles más pobres tienen una representación del 2 y $3 \%$ respectivamente (MENDES BRAGA, 2008).

11 Este temática se abordó especialmente en Chiroleu (2009). 


\section{EVALUACIÓN INSTITUCIONAL SIN MEJORAMIENTO DE LA CALIDAD DE LOS APRENDIZAJES}

Los años 90 supusieron la introducción en América Latina de un conjunto de políticas que transformaron profundamente a la educación superior. En este sentido el cambio de la relación Estado-Universidades y el establecimiento de mecanismos de evaluación institucional como forma de regulación, pusieron a la calidad en el centro de la escena.

En el mismo lapso, pero con ritmos y profundidades diversas, los países de la región fueron suscribiendo las políticas y el lenguaje de época y se fue generando un sentido común evaluativo que penetró profunda y capilarmente en las instituciones y en menor medida, en las prácticas académicas. La evaluación no es un dispositivo ajeno al ámbito educativo y la meta de elevar la calidad académica afectada por la masificación sin generación de condiciones institucionales y presupuestarias para afrontarla, era un objetivo no desdeñable; esto se asoció a gobiernos con una fuerte voluntad política de intervención en el sector y generosos créditos internacionales provenientes de las mismas usinas que proponían la reforma.

El resultado final fue un proceso que instaló y legitimó la evaluación institucional como un fin en sí mismo, perdiendo a menudo de vista que constituye (y debe necesariamente constituir) una herramienta para el mejoramiento de la calidad. Por otra parte, se tendió a ignorar la existencia de diversas perspectivas en torno a la definición, los alcances y los fines de la calidad, y a operar sin reconocer el carácter contingente de la política pública, esto es, la necesidad de adaptarla a las condiciones puntuales generadas en un momento y en un lugar determinado. ${ }^{12}$

$\mathrm{Al}$ respecto, puede reconocerse la existencia de dos vertientes principales ${ }^{13}$ en términos de abordaje del proceso evaluativo: una que lo liga al mejoramiento de la calidad y lo asume como un acto autónomo de la institución orientado por un objetivo emancipatorio, y otra, que persigue el aseguramiento de la calidad y que se centra en el cumplimiento por parte del estado de una función regulatoria y de control centralizado y burocrático del funcionamiento del sector $^{14}$. Esta última es la que mayoritariamente ha prevalecido en la medida en que constituye una herramienta privilegiada para facilitar el ingreso del mercado en el ámbito de la educación superior y con él, la instalación de una

$12 \mathrm{Al}$ respecto puede consultarse Stein, Tomassi, Echebarría, Lora y Paine (2006).

13 Para una revisión exhaustiva de las diversas concepciones de calidad puede consultarse Fernández Lamarra (2007).

14 Sobre este punto pueden consultarse entre otros, Stubrin (2008), Rothen (2006) y Dias Sobrinho (2002). 
lógica eficientista mucho más vinculada a pautas económicas que a necesidades educativas (DIAS SOBRINHO, 2007, p. 321).

El saldo común fue el establecimiento en la mayoría de los países de algún tipo de evaluación, pero el concepto de calidad al que ésta se vincula y la intensidad con que el proceso permeó a las instituciones está ligado entre otras cuestiones, a las características y grado de movilización de sus actores y a la generación por parte de los gobiernos de políticas activas sobre la temática. ${ }^{15}$

En el caso argentino ${ }^{16}$, por ejemplo, la evaluación ingresa tempranamente en la agenda de gobierno de C. Menem y es fuertemente resistida por diversos actores universitarios. Esto hace que cuando en 1995 se crea la Comisión Nacional de Evaluación y Acreditación Universitaria (CONEAU) se llegue a una solución de compromiso que procura reconocer las singularidades institucionales en vez de construir modelos de validez nacional. En este sentido, se establece que las instituciones desarrollarán procesos de autoevaluación institucional para analizar los logros y dificultades en el cumplimiento de sus funciones y sugerir medidas para su mejoramiento y que las evaluaciones externas se inscribirán en el marco de los objetivos definidos por cada institución. Estos procesos por su parte, no están articulados con la obtención de fondos complementarios ni financiamientos especiales y el objetivo general es el mejoramiento de la calidad tratando de orientar las metas a alcanzar a los objetivos institucionales.

Luego de más de quince años de funcionamiento, el peso burocrático y las capacidades estatales instaladas por la CONEAU han consolidado las tareas de evaluación, lo cual conduce -en términos de Camou- a un equilibrio regresivo en el que los actores involucrados en el proceso ritualizan sus conductas y las propias prácticas evaluativas, vaciándolas de contenido. Se da un bloqueo de la política de evaluación la cual navega entre la burocratización de los evaluadores, la falta de compromiso de los actores universitarios y del propio gobierno (CAMOU, 2007).

Esta ritualización del proceso evaluativo le quita sentido y conspira contra el objetivo de mejoramiento de la calidad que le dio origen y justifica su existencia. Lejos de conformarse en las instituciones una cultura de la evaluación que potencie un comportamiento proactivo de sus actores, el desarrollo de los

15 Sin embargo, por efectos de la globalización se producen muchas convergencias entre las principales tendencias internacionales y las experiencias locales (DIAS SOBRINHO, 2007, p. 311).

16 Para los casos de Argentina y Brasil, un tratamiento más extenso puede encontrarse en (CHIROLEU, A. et al, 2009). En este trabajo se da cuenta de los orígenes de la evaluación en el país y del fracaso del intento por imponer un proyecto más ortodoxo, próximo a las propuestas de los organismos multilaterales de crédito. 
procesos se asocia a la necesidad de cumplir con la normativa desde un punto de vista meramente formal.

A mediados de los años 90, en Brasil por su parte, con la premisa de garantizar la calidad del sistema que se había expandido fuertemente a través de su componente privado, se establece el Examen Nacional de Cursos, conocido popularmente como Provâo, el cual evalúa los contenidos adquiridos en el transcurso de las carreras de grado. Se trata de evaluaciones individuales que luego pasan a ser consideradas evaluaciones de las carreras y de las instituciones, conformándose -a partir de esta información- rankings de establecimientos que inciden en las estrategias de selección de instituciones por parte de los alumnos y sus familias. ${ }^{17} \mathrm{Al}$ respecto es bien sabido -señala Dias Sobrinho (2002)- que los rankings no generan efectos educativos, pero sí lo hacen en términos de mercado

A partir de 2003, en la gestión de Lula da Silva, la evaluación conserva un lugar central en las políticas de educación superior; se suceden entonces dos etapas que dan cuenta de la puja entre grupos portadores de visiones opuestas sobre la función de la evaluación. En este sentido en los primeros meses de la gestión se constituye la Comisión Especial de Evaluación (CEA), encargada de efectuar un diagnóstico de la situación y de reformular los procesos, instrumentos y políticas de evaluación y regulación. Constituida principalmente por académicos, esta comisión se propone recuperar la dimensión emancipatoria de la evaluación que articulara su aspecto formativo con la regulación del sistema ${ }^{18}$.

Sin embargo, la Ley 10.861/04 que debía recoger aquella propuesta, surge del compromiso con grupos y lobbies del sector y procura transformar los rasgos más criticados del Provâo sin variar sustancialmente los criterios establecidos en el transcurso del gobierno de Cardoso. El SINAES (Sistema Nacional de Evaluación de la Educación Superior) consta de tres ejes: la evaluación institucional, que incluye una autoevaluación de las propias instituciones y una evaluación externa a cargo de una oficina gubernamental, la evaluación de las carreras y la evaluación de los estudiantes, que incluye a los ingresantes y a los que se gradúan.

17 A pesar de sus deficiencias técnicas, señala Barreyro (2006) el Provâo se constituyó en una herramienta útil para el cumplimiento de la respectiva política por su capacidad para comunicar de manera sencilla los resultados de las evaluaciones. En este sentido, aunque las carreras no pueden ser comparadas entre sí por sus disímiles características o grado de dificultad, la asignación de una calificación de "A", "B", "C", "D" o "E" generó la posibilidad de conformar rankings de instituciones.

18 En el documento presentado por la CEA se procura separar la idea de evaluación de la de regulación, destacando que se trata de aspectos diversos, y que el segundo no es consecuencia del primero (ROTHEN, 2006). 
Se trata de un complejo procedimiento -que ha merecido fuertes críticas desde el punto de vista técnico y político ${ }^{19}$ - y que conduce nuevamente a la elaboración de rankings de instituciones y a la pérdida de la globalidad de la propuesta, la que se centra una vez más en una organización jerárquica de las instituciones, útil para orientar la demanda del mercado, pero incapaz de reflejar los matices del sistema.

En el caso de México, la evaluación constituye sin duda el eje de la reforma de los 90; la misma se institucionalizó a través del Programa para la Modernización Educativa (1989-1994) ejecutado por el Gobierno Federal; la CONAEVA (Comisión Nacional para la Evaluación de la Educación Superior) organiza el marco de funcionamiento del Sistema Nacional de Evaluación (autoevaluación, evaluación del sistema y evaluación interinstitucional), el cual se pone en marcha en los años sucesivos. En 1994 se crea el CENEVAL (Centro Nacional de Evaluación para la Educación Superior) cuyo objetivo es "contribuir a mejorar la calidad de la educación media superior y superior evaluando los aprendizajes logrados en cualquier etapa de los procesos educativos mediante la aplicación de exámenes estandarizados" (RUBIO OCA, 2006). ${ }^{20}$

En estos años de trabajo continuo la evaluación se ha ido consolidando, conformándose una "cultura de la evaluación", según lo reconoce el propio Programa Nacional de Educación 2001-2006. Sin embargo, diversos actores señalan la existencia de numerosos puntos críticos en este proceso. Uno de ellos es el número de instancias de evaluación a cubrir por los académicos en términos individuales e institucionales, las cuales, al estar a menudo ligadas al financiamiento, son completadas de manera compulsiva y acrítica, restándole densidad al proceso mismo (DÍAZ BARRIGA, 2005). ${ }^{21}$

Por otra parte, la participación de México en el Tratado de Libre Comercio con América del Norte y en la OCDE genera una acentuada internacionalización de su educación superior y una mayor exigencia por parte de sus socios para la adopción de criterios de validación y competitividad de sus instituciones y diplomas de educación superior.

En Venezuela, la evaluación institucional ingresa en la agenda a partir de 1995 de la mano del Consejo Nacional de Universidades coincidiendo con los

$19 \mathrm{Al}$ respecto pueden consultarse De Moura Castro (2008) y Barreyro (2008).

20 Se aplican exámenes nacionales para el ingreso a la educación media superior, superior (técnico superior universitario, profesional asociado y licenciatura) y posgrado; también se evalúa a los alumnos que han cubierto un tramo de la licenciatura, así como a los egresados de una carrera de técnico superior universitario o licenciatura (RUBIO OCA, 2006).

21 Luis Porter hace por ejemplo referencia a "la universidad de papel" dando cuenta del peso de las demandas burocráticas sobre la universidad en términos de informes a completar que se suceden ocupando el espacio de las tareas productivas de generación de conocimiento. 
alcances que el tema tenía en el resto de la región. Sin embargo, no pasó del plano declarativo, pues no se generaron avances significativa en la medida que el organismo a cargo era el que nucleaba a los rectores, actores poco interesados en poner en juego sus bases de sustentación (ZIRITT; OCHOA HENRÍQUEZ, 2008).

Luego de otros intentos fallidos, en 2008 se constituyó el Comité de Evaluación y Acreditación de Programas e Instituciones de Educación Superior (CEAPIES), órgano integrado por académicos de reconocida trayectoria, cuya función es coordinar los procesos de evaluación y acreditación institucional, para garantizar, reconocer y promover la calidad de la educación universitaria. Este Comité ha operado especialmente para lograr la integración de Venezuela a la Red de Agencias Nacionales de Acreditación del Mercosur (RANA) y a la Red Iberoamericana de Acreditación y Evaluación de la Educación Superior (RIACES). Asimismo, ha coordinado los procesos de evaluación y acreditación de las carreras de Agronomía y Arquitectura en el marco del Sistema de Evaluación y Acreditación de Programas Universitarios (ARCUSUR) del Mercosur (MINISTERIO DEL PODER POPULAR PARA LA EDUCACIÓN SUPERIOR, 2010).

Desde la CEAPIES se sostiene que "la calidad de la educación tiene que ver con el grado de compromiso de los programas e instituciones con el desarrollo soberano de la patria socialista, por lo que es un reto desafiar la concepción tradicional que sobre este particular se tiene". La intención es "impulsar los procesos de evaluación y acreditación en el marco del Alba, Unasur, de la Organización de Estados Latinoamericanos y Caribeños, entre otros, con el claro objetivo de generar las condiciones subjetivas y materiales para que el subsistema esté imbuido en la direccionalidad estratégica de la Revolución Bolivariana Socialista".

En este caso, como puede apreciarse, en las últimas dos décadas se sucedieron diversos mecanismos que no fueron exitosos en desarrollar procesos continuos de evaluación. La experiencia actual es muy reciente por lo cual se hace difícil analizar sus logros, los cuales aparecen además ligados a facilitar la integración regional de Venezuela. Sus bases de funcionamiento sin embargo, se encuadran una vez más en criterios extraacadémicos y extrainstitucionales, poniéndose al servicio de un proyecto político.

Cabría preguntarse: la aplicación de estas políticas sin la construcción de consensos amplios $\mathrm{y}$, a menudo, sin la incorporación de recursos económicos suficientes para encarar las transformaciones necesarias ¿mejoró la calidad de la educación superior? Existen pistas que, al menos, introducen dudas al respecto, lo cual es especialmente grave en la medida en que estos procesos insumen 
considerables recursos y energías, que se apartan de las actividades y prácticas habituales para cumplir con las crecientes y constantes demandas ejercidas por las instancias de evaluación que se han rápidamente burocratizado.

Otro interrogante focaliza en la pertinencia de aplicar - con fines regulatorios- metodologías evaluativas surgidas en otros contextos institucionales y sistémicos muy diversos, que condujeron en el ámbito regional, a prácticas ritualistas que no operaron transformaciones virtuosas. En este punto resulta conveniente considerar que los modelos son contingentes en tiempo y espacio por lo que su aplicación no garantiza iguales resultados urbis et orbis y además, la experiencia en América Latina muestra el reiterado fracaso que supone ignorar este principio.

En esta situación general, la expansión compulsiva de los estudios de posgrado y el énfasis en el crecimiento del segmento de doctores, tampoco parece tener incidencia favorable en el mejoramiento de la formación de los estudiantes y la producción de conocimiento.

Esto no implica proponer la eliminación de los procesos de evaluación y / o acreditación, sino, en todo caso, efectuar un análisis desapasionado de sus logros y zonas grises que permita aprovechar sus potencialidades, reducir sus limitaciones y sobre todo, orientarlo hacia procesos efectivos de mejora de la calidad.

\section{PRIMACÍA DEL PROFESIONALISMO POR SOBRE EL COMPROMISO Y LA RESPONSABILIDAD SOCIAL UNIVERSITARIA}

En América Latina, la universidad colonial tuvo como características axiales, su aislamiento del entorno social y un sesgo fuertemente profesionalista que perduró y se reprodujo con el transcurso del tiempo, y que puede graficarse -en términos de Hans Steger- a través de la denominada "universidad de los abogados". Institución formadora de profesionales al margen de las necesidades de la sociedad que los cobijaba, actuaba en este sentido como legitimadora del orden establecido, otorgando a través de sus diplomas, un instrumento de distinción en el contexto de una sociedad en formación.

Es la Reforma Universitario de 1918 la que le reclama un relacionamiento más estrecho con su entorno, planteado por entonces a través de la figura de la extensión universitaria que procuraba profundizar los lazos entre la institución y la sociedad. Su mérito entonces, consistió en advertir que más allá de las funciones clásicas de docencia e investigación, existía otra, de carácter 
social, tan importante e ineludible como aquellas, especialmente atendiendo a las características y déficit socioeconómicos de la región, en cuya atención, la universidad debía intervenir.

Esta propuesta centrada en la proyección hacia la sociedad de la labor universitaria supuso por entonces una importante ruptura que buscó dinamizar el funcionamiento institucional, ampliando sus horizontes; sin embargo, este proceso tendió a naturalizarse y a generar prácticas que, a pesar de expresar a menudo intenciones solidarias, reforzaron el individualismo y el aislamiento institucional con relación a los requerimientos sociales.

Se atribuye a Paulo Freire ${ }^{22}$ la inauguración de una segunda etapa en la reflexión sobre la temática, a partir del reconocimiento que la comunicación universidad-sociedad debe constituir una calle de doble mano, con influencias recíprocas y un proceso de construcción y resignificación de los lazos mutuos, posición que conduce a lo que en la actualidad se denomina responsabilidad social universitaria (RSU).

Este concepto tiene su origen en el mundo empresarial y es posteriormente aplicado en otros ámbitos, procurándose asimismo su adaptación a las singularidades de la organización universitaria. Se trata de una propuesta interesante y fecunda que se extiende de manera disímil en el entramado de las instituciones de la región, contando con proyectos activos en algunos establecimientos de varios países, mientras en otros, sus fundamentos recién comienzan a ser discutidos.

Vinculada estrechamente con las necesidades de la región, la propuesta se sustenta en la voluntad de que las universidades constituyan un agente privilegiado de transformación social a través de la formación de sus graduados en normas éticas y valores que incentiven un compromiso social que priorice la atención de los sectores más desfavorecidos (TÜNNERMANN BERNHEIM, 2010, p. 19).

La RSU procura resignificar las tradicionales tareas de servicio social universitario privilegiando su sentido transformador que se pone al servicio del mejoramiento de la formación y producción de conocimientos. Se trata de un modo de gestión integral de los impactos humanos, sociales y ambientales que, siguiendo a Vallaeys, puede definirse como una estrategia de gerencia ética e inteligente de los impactos que genera la institución en su entorno. Constituye de tal modo, una conducta ética que motoriza a la organización permitiéndole avanzar más allá de lo estrictamente demandable, desarrollando y exigiendo conductas de corresponsabilidad con el medio (TORRES PERNALETE; TRÁPAGA ORTEGA, 2010, p. 50-77).

$22 \mathrm{Al}$ respecto puede consultarse Tünnermann Bernheim (2010). 
Documentos el BID -institución que apoya intensamente la propuesta- sostienen que no supone una acción social filantrópica como actividad marginal en la institución, sino un nuevo sistema de gestión de la organización que opera en todas sus funciones a partir de un diagnóstico y de la gestión de los impactos. Constituye así, una manera de asegurar la sostenibilidad social y ambiental de la producción y consumo humano. Implica la buena gobernabilidad, esto es la definición de la misión de la institución y un código de ética que proteja los derechos fundamentales de las personas; la gestión de los impactos ambientales y sociales; la permanente rendición de cuentas a los interesados involucrados y la intermediación que permita acercar intereses complementarios con el objetivo final de lograr un desarrollo sostenible (VALLAEYS; DE LA CRUZ; SASIA, 2009).

Asociada al concepto de pertinencia social, su gestión debe inscribirse en la misión, objetivos y funciones de sus instituciones, en lo atinente al cumplimiento de lo que la sociedad actual está demandando; en este sentido, las universidades deben buscar alternativas que les permitan convertirse en intérpretes críticos de sí mismas (TORRES PERNALETE; TRÁPAGA ORTEGA, 2010, p. 80).

Si el objetivo es alcanzar un Desarrollo humano sustentable, resulta indispensable una transformación de las bases mismas del trabajo académico que permita la formación de los estudiantes y la generación de conocimiento a partir de criterios de sensibilidad social y de atención de las demandas de la comunidad. Esto supone una transformación profunda de las funciones de docencia e investigación y de la propia gestión de las instituciones, única vía para romper el aislamiento y el desentendimiento de las necesidades sociales ${ }^{23}$.

Existen sin embargo diversas resistencias y obstáculos que atentan contra una generalización de esta práctica. Entre ellos, Torres Pernalete y Trápaga Ortega (2010, p. 81) señalan las contradicciones históricas de la sociedad en que las instituciones se encuentran inmersas, que redunda en su comportamiento fuertemente influenciado por lo socioeconómico, lo político y lo cultural ${ }^{24}$. Al respecto, Vallaeys (2008) sostiene la necesidad de reconocer que ninguna institución es éticamente neutral por lo que -de manera consciente o a través del currículo oculto ${ }^{25}$ - tiende a legitimar ciertos comportamientos y hábitos de

23 En este sentido, Vallaeys (2008) sostiene que la RSU exige articular las diversas partes de la institución en un proyecto de promoción social de principios éticos y de desarrollo social equitativo y sostenible, para la producción y transmisión de saberes responsables y la formación de profesionales-ciudadanos igualmente responsables.

24 En este sentido, en opinión de Bok (2009, p. 23) las universidades han experimentado históricamente dificultades para conciliar las demandas prácticas y urgentes del público, con los intereses a largo plazo de la institución académica.

25 El concepto de "currículo oculto" constituye una herramienta útil para reconocer en qué medida la 
vida. Precisamente de la discusión en torno a estos factores, frecuentemente naturalizados, que fijan una impronta indeleble en la formación de los graduados universitarios, pueden surgir vías de reconocimiento y de transformación del quehacer universitario.

Por su parte, Carrizo (2011) opina que la amplitud y diversidad de las prácticas que abarca el concepto de RSU, atenta contra la precisión del mismo y su correcta aplicación. Resulta indispensable sin embargo, señalar que un elemento clave para la aplicación del concepto es la construcción de sólidos consensos institucionales y la discusión profunda de la misión, los objetivos y las metas de las instituciones de cara a los requerimientos regionales y el desarrollo humano sustentable.

Como señaláramos anteriormente, este concepto ha sido sustentado por el BID en el contexto de la Iniciativa Interamericana de Capital Social, Ética y Desarrollo, especialmente a través de la Red Universitaria de Ética y Desarrollo social. Las experiencias que se desarrollan en toda América Latina son sin embargo, de envergadura diversa, aunque en líneas generales se encuentran aún en un estado embrionario ${ }^{26}$, en el que prima la conveniencia de la instalación del debate y el avance paulatino en la transformación de las prácticas.

Por otra parte, existe una relación de complementariedad entre extensión y RSU, en la cual esta última actúa como eje organizador de todas las funciones universitarias avanzando de tal manera en la construcción de un modelo educativo con mayor pertinencia social (TORRES PERNALETE; TRÁPAGA ORTEGA, 2010, p. 84-85). De lo que se trata en última instancia es de lograr un cambio virtuoso de aquella, que ancle en los programas de estudio y las formas de evaluación de los estudiantes: esto es, superar la condición marginal que habitualmente ha alcanzado la extensión universitaria para dotarla de una centralidad que aproxime a la institución a la sociedad. En este sentido, en opinión de Vallaeys (2008), un correcto uso de la proyección social y la extensión universitaria permitirá acercar soluciones a los problemas éticos de la enseñanza universitaria, cuando se quiere realmente enseñar a través de proyectos de desarrollo.

De tal manera, la formación ética y socialmente responsable ya no puede ser entendida como un "complemento deseable" de la formación profesional, sino como un eje de valor de las competencias especializadas del egresado

enseñanza académica participa y refuerza, de modo muchas veces inconsciente, "la reproducción de las injusticias y patologías del mundo actual, participación que sólo se puede negar desde la ingenua y falsa autonomización de la institución educativa de su contexto social" (VALLAEYS, 2008).

26 Para un pormenorizado detalle de las experiencias en América Latina, puede consultarse Torres Pernalete y Trápaga Ortega (2010, p. 51-75). 
universitario y una condición de posibilidad de la pericia e integralidad del nuevo profesional (CARRIZO, 2011).

En este mismo sentido, aunque sin utilizar el concepto de RSU, Boaventura de Sousa Santos (2005) sostiene que resulta impensable una universidad pública que no cumpla de manera simultánea con las tres funciones básicas: docencia (de grado y posgrado), investigación y extensión. Sin embargo, las mismas deben además orientarse a la democratización del bien público universitario, es decir, constituir una contribución específica de la universidad en la definición y solución colectiva de los problemas sociales, nacionales y globales. La reforma de la universidad debe conferir una nueva centralidad a las actividades de extensión y concebirlas de modo alternativo al capitalismo global, atribuyendo a las universidades una participación activa en la construcción de sociedades más integradas, en la profundización de la democracia y en la defensa de la diversidad cultural.

Las tendencias que a nivel regional y planetario presenta el desarrollo universitario parecen estar sin embargo, bastante alejadas de estas propuestas. Por una parte, una primera luz de alarma se enciende cuando observamos la gran heterogeneidad de los sistemas de educación superior y el peso específico que en los mismos adquiere el segmento privado con fines de lucro. Al respecto cabe interrogarse sobre la viabilidad que instituciones de este tipo se interesen por desarrollar proyectos de RSU.

Por otra parte, en el plano académico, la educación clásica y humanista tiende a ser reemplazada por el vocacionalismo que prioriza el entrenamiento para el mercado de trabajo mientras la tradicional enseñanza enciclopédica liberal, es reemplazada por un modelo científico-tecnológico que se basa en tecnología proveniente de los países centrales (SCHUGURENSKY, 2002). Este vocacionalismo se presenta así como un nuevo universalismo y la $\mathrm{em}$ presa -considerada el modelo "ideal" de organización universitaria- aparece como el modelo organizacional para estructurar prácticas, valores y rutinas que corporiza esta nueva universalidad. La empresa no sólo orienta los procesos de formación e investigación sino que reemplaza la tradicional concepción de la universidad como espacio público y lugar de reflexión y controversia entre muchas identidades (KROTSCH, 2001).

De esta manera, la educación deja de ser un fin en sí mismo y procura lograr una estrecha articulación entre sus contenidos curriculares y las demandas del mercado de trabajo, ignorando los efectos disruptivos -en términos sociales y educativos- que esta mirada cortoplacista puede tener y los cambios vertiginosos que aquel ha experimentado en las últimas décadas. Esto incentiva a los estudi- 
antes a desarrollar una visión pragmática de la educación que pone el acento en la tasa de retorno a obtenerse, desplazando sus efectos en términos de formación de pensamiento autónomo y crítico y responsabilidad social, desperdiciando así la posibilidad de aprovechar las potencialidades de las universidades para la construcción de sociedades menos desiguales y más integradas.

Queda pendiente de respuesta la pregunta en torno a las reales posibilidades que existen de contrarrestar estas tendencias dominantes en lo que respecta a la formación de profesionales, la defensa de intereses personales y particularistas y el utilitarismo pragmático en un contexto general poco propicio caracterizado por la primacía de un individualismo corrosivo y conductas hedonistas.

\section{LA PERSISTENCIA DE LOS VIEJOS PROBLEMAS: ¿CUESTIONES INSOLUBLES O RECETAS INAPROPIADAS?}

En esta presentación abordamos un conjunto de problemas de la educación superior de la región, que aunque tienen vigencia al menos desde la constitución de los sistemas, no por ello han perdido actualidad. Muchas otras cuestiones tienen sin embargo un status similar: los alcances actuales de la autonomía, la expansión anárquica de los sistemas, la producción de conocimiento alejado de las necesidades nacionales y regionales, la difícil inserción de los graduados en la estructura ocupacional, etc.

En muchas de estas cuestiones puede apreciarse un déficit de políticas públicas de educación superior acompañado a menudo por una mora institucional en la atención de estas problemáticas. En lo que respecta al primer nivel, cabe insistir en las singularidades propias del campo de la educación superior, un ámbito especialmente sensible que históricamente ha merecido en los diversos países, formas alternativas de atención de la demanda por parte de los estados.

Llama la atención la continuidad en varios de los países de la región de las políticas de educación superior esbozadas y aplicadas en los 90, más allá de la alternancia entre gobiernos de corte neoliberal y gobiernos de otro cúneo (en algunos casos, considerados "progresistas"), lo que parecería dar cuenta, una vez más, tanto de la penetración en la región de las propuestas provenientes de los considerados "modelos de éxito" en el desarrollo del sector, como de las dificultades para consensuar y generar propuestas alternativas más afines a las problemáticas locales. Dentro de los casos analizados, la única excepción la plantea Venezuela, país cuya educación superior, si bien no había sido profundamente penetrada por las reformas de los 90 , experimenta además un cambio de paradigma propuesto por sus gestores políticos. Se trata de un caso 
interesante para observar y efectuar una evaluación integral de las políticas aplicadas, lo cual sólo será posible dentro de algún tiempo.

En líneas generales, la persistencia de los viejos problemas aún teniendo en cuenta las singularidades de la relación estado/gobierno-universidades supone pues, una doble dificultad: ni los gobiernos (independientemente de su signo) han generado las políticas públicas acordes para enfrentarlos, ni las instituciones y sus actores han sabido o podido repensarse a sí mismos y sus prácticas para generar soluciones acordes al desafío planteado. En este sentido, los académicos y las autoridades institucionales de varios países, han tendido a enfrentar las políticas oficiales a través de respuestas rituales y vacías de contenido: esto, que se aprecia -especial pero no exclusivamente- en la aplicación de procesos de evaluación institucional, implica una pérdida de energías y de recursos, poco acorde a las penurias económicas expresadas en la mayoría de los países.

Cabe sin embargo destacar una cuestión esperanzadora: la emergencia de nuevas políticas cuya aplicación podría incidir en una transformación más endógena y más ligada a pautas de desarrollo sustentable. Al respecto si bien las políticas de inclusión y la RSU están aún desigual y escasamente desarrolladas en los países y los establecimientos, pueden constituir vías adecuadas para una movilización virtuosa de nuestras instituciones y sistemas.

En un clima de mayor tolerancia hacia la diversidad como el propio a esta primera década del siglo XXI, resulta factible plantear ciertos temas que hubieran sido impensables hace apenas un par de décadas. Este es el caso, entre otros, de la necesidad de generar condiciones equitativas de ingreso, permanencia y egreso para todos los grupos sociales y de poner a la universidad al servicio de las necesidades y requerimientos de las respectivas sociedades. En ambos casos, la realidad de América Latina y los intensos mecanismos de desigualdad que en ella operan, dan cuenta de la premura y centralidad de generar prácticas alternativas, así como de los escollos que las mismas deberán sortear.

$\mathrm{Al}$ respecto, si bien se ha dado un importante avance a través del reconocimiento de las problemáticas, falta aún recorrer un largo camino. Y en este sentido cabe alertar sobre la necesidad de operar a partir de la construcción de sólidos consensos institucionales para que la aplicación de este tipo de políticas no genere -una vez más- comportamientos reactivos y/o rituales que anule o limite su potencial democratizador a través de la conformación de respuestas formales, carentes de efectos concretos.

Si durante décadas, pero especialmente en los últimos años, la adopción de los modelos considerados exitosos a nivel internacional se ha mostrado estéril para resolver estos nudos gordianos y, a esta altura existen certezas que las viejas 
recetas no resultan apropiadas, cabe introducir nuevas visiones y modalidades que permitan una renovación profunda de las prácticas: no es que los problemas sean insolubles, sino que los mapas que utilizamos son inadecuados.

La tarea no es sencilla, y convoca a los hacedores de política a nivel nacional e institucional a demostrar su solvencia en la elaboración de una agenda local y regional de educación superior que brinde posibilidades de pensar su devenir desde una perspectiva que, mirando hacia adentro para poder luego (y sólo luego) mirar hacia afuera, dé solución a los déficit de inclusión social y política, mejore la calidad de las instituciones y los aprendizajes y acreciente la responsabilidad social con el entorno, únicas vías de responder al reto de nuestros días.

\section{REFERENCIAS}

ACOSTA SILVA, Adrián. El neointervencionismo estatal en la Educación Superior de América Latina. Sociológica, Azcapotzalco, México-DF, v. 7, n 49, p. 43-72, 2002.

APONTE HERNÁNDEZ, Eduardo. Desigualdad, Inclusión y Equidad en la Educación Superior en América Latina y el Caribe: Tendencias y escenario alternativo en el horizonte 2021. In: GAZZOLA, Ana Lúcia; DIDRIKSON, Axel. (Ed.). Tendencias de la educación superior en América Latina y el Caribe. Caracas: IESALC-UNESCO, 2008. p. 113-154.

BARREYRO, Gladys Beatriz. De exames, rankings e mídia. Avaliação, Campinas; Sorocaba, v. 13, n. 3, 2008.

BARREYRO, Gladys Beatriz. Evaluación de la educación superior brasileña. El SINAES. Revista de la Educación Superior, México-DF, v. 25, n. 137, 2006.

BOK, Derek. Más allá de la Torre de Marfil. La responsabilidad social de la universidad moderna. Buenos Aires: Universidad de Palermo, 2009.

BRAGA, Mauro Mendes. Inclusão e equidade: desafios para a educação superior na América Latina e no Caribe na próxima década. In: GAZZOLA, Ana Lúcia; DIDRIKSON, Axel. (Ed.). Tendencias de la educación superior en América Latina y el Caribe. Caracas, IESALC-UNESCO, 2008. 
CAMOU, Antonio. Los juegos de la evaluación universitaria en la Argentina. Notas sobre las interacciones conflictivas entre Estado y Universidad. In: KROTSCH, Pedro; CAMOU, Antonio; PRATI, Marcelo. (Comp.). Evaluando la evaluación. Políticas universitarias, instituciones ya ctores en Argentina y América Latina. Buenos Aires: Prometeo Libros, 2007.

CARRIZO, Luis, Sed de ética, capital social y desarrollo en América Latina. [s.d.] Disponible en: <http://www.udlap.mx/rsu/pdf/3/ SeddeEticaenAmericaLatina.pdf>. Consultado el: 24 jan. 2011.

CASTRO, Claudio de Moura. Quem entendeu a nova avaliação do ensino? Veja, São Paulo, n. 20079, 2008.

CHIROLEU, Adriana. Políticas públicas de inclusión en la educación superior Los casos de Argentina y Brasil. Pro-Posições, Campinas, v. 20, n. 2, p. 141-166, maio/ago. 2009.

CHIROLEU, Adriana; IAZZETTA, Osvaldo. La política universitaria en la agenda de gobierno de Kirchner. In: MARQUINA, Mónica, MAZZOLA, Carlos; SOPRANO, Germán. (Comp.). Políticas, instituciones y protagonistas de la universidad argentina. Buenos Aires: UNGS, UNSL y Prometeo Libros, 2009. p. 21-46.

DIAS SOBRINHO, José. Educacao e avaliacao: técnica e ética. In: DIAS SOBRINHO, José; RISTOFF, Dilvo. (Comp.). Avaliacao democrática para uma universidade cidadã. Florianopolis: Insular, 2002.

DIAS SOBRINHO, José Evaluación de la educación superior en Brasil: la cuestión de la calidad. In: KROTSCH, Pedro; CAMOU, Antonio; PRATI, Marcelo. (Comp.). Evaluando la evaluación. Políticas universitarias, instituciones ya ctores en Argentina y América Latina. Buenos Aires: Prometeo Libros, 2007.

DIAZ BARRIGA, Angel. Riesgos de los Sistemas de Evaluación y Acreditación de la Educación Superior. Seminario Regional: Las nuevas tendencias de la Evaluación y Acreditación en América Latina y el Caribe. Buenos Aires: CONEAU, 2005.

DIDRIKSON, Axel. Contexto global y regional de la Educación en América Latina y el Caribe. In: GAZZOLA, Ana Lúcia; DIDRIKSON, Axel. (Ed.). Tendencias de la educación superior en América Latina y el Caribe. Caracas, IESALC-UNESCO, 2008. 
FERNÁNDEZ LMARRA, Norberto. Educación superior y calidad en América Latina y Argentina. Los procesos de evaluación y acreditación. Buenos Aires: IESALC/UNESCO/EDUNTREF, 2007.

KROTSCH, Pedro. La dilusión de las nociones de misión e idea de la universidad moderna: el papel de las demandas o finalidades asignadas. In: NAISHTAT, Francisco; GARCÍA RAGGIO, Ana; VILLAVICENCIO, Susana. (Comp.). Filosofías de la universidad y conflicto de racionalidades. Buenos Aires: Colihue, 2001.

MINISTERIO DEL PODER POPULAR PARA LA EDUCACIÓN SUPERIOR. La Revolución Bolivariana en la Educación Universitaria 1999-2009. Caracas: Gobierno Bolivariano de Venezuela, 2010.

ORDORIKA, Imanol. Educación Superior y Globalización: las universidades públicas frente a una nueva hegemonía. Revista Andamios, México-DF, v. 3, n. 5, 2006.

ROTHEN, José Carlos. Ponto e contraponto na Avaliação Institucional. Análise dos documentos de implantação do SINAES. In: SILVA Jr., Joao dos Reis; OLIVEIRA, Joao Ferreira de; MANCEBO, Deisi. (Comp). Reforma universitária: dimensões e perspectivas. Campinas: Alínea-Anped, 2006.

RUBIO OCA, Julio. La política educativa y la educación superior en México 1990-2006. México DF: SEP - FCE, 2006.

SANTOS, Boaventura de Souza. La universidad en el siglo XXI. Para una reforma democrática y emancipadora de la universidad. Buenos Aires: Laboratorio de Políticas Públicas y Miño y Dávila, 2005.

SCHUGURENSKY, Daniel. Autonomía, heteronomía y los dilemas de la educación superior en la transición al siglo 21: el caso Canadá. In: RODRÍGUEZ GÓMEZ, Roberto. (Comp.). Reformas en los sistemas nacionales de educación superior. Coruña: Netbiblo-RISEU, 2002.

STUBRIN, Adolfo. Los mecanismos nacionales de garantía pública de calidad en el marco de la internacionalización de la educación superior. In: DIAS SOBRINHO, José; RISTOFF, Dilvo; GOERGEN, Pedro. (Org.). Universidade e sociedade: perspectivas internacionais. Sorocaba: Eduniso, 2008. 
TORRES PERNALETE, Mariela; TRÁPAGA ORTEGA, Miriam. (Org.). Responsabilidad social de la universidad. Retos y perspectivas. Buenos Aires: Paidós, 2010.

TÜNNERMANN BERNHEIM, Carlos. Prólogo. In: TORRES PERNALETE, Mariela; TRÁPAGA ORTEGA, Miriam. (Org.).

Responsabilidad social de la universidad: retos y perspectivas. Buenos Aires: Paidós, 2010. p. 236

VALLAEYS, François. ¿Qué es la responsabilidad social universitaria? 2008. Disponible en: <www.ausjal.org/files/rsu.doc >. Consultado el: 24 jan. 2011.

VALLAEYS, François; DE LA CRUZ, Cristina; SASIA, Pedro.

Responsabilidad social universitaria. Manual de primeros pasos. México: McGraw Hills Editores y BID, 2009.

ZIRITT, Gertrudis; OCHOA HENRÍQUEZ, Haidée. La reforma universitaria en Venezuela: promovida por el Consejo Nacional de Universidades en los 90. Laurus, Caracas, v. 14, n. 26, p. 31-55, enero-abr., 2008. 